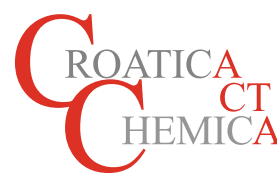

\title{
Electrofugality of Some Ferrocenylphenylmethyl Cations
}

\author{
Sandra Jurić, Marijan Marijan, Olga Kronja*
}

University of Zagreb, Faculty of Pharmacy and Biochemistry, Ante Kovačića 1, HR-10000 Zagreb, Croatia
* Corresponding author's e-mail address: okronja@pharma.hr

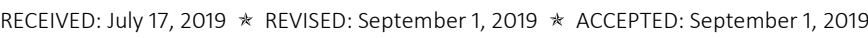

This PAPER IS DEDiCATED to Prof. KATA MLINARIĆ-MAJERSKI ON THE OCCASION OF HER 70 ${ }^{\text {Th }}$ BIRTHDAY

\begin{abstract}
The electrofugality scale has been extended with new substituted ferrocenylphenylmethyl cations 1-4. $E_{\mathrm{f}}$ values were determined by applying the linear free energy relationship (LFER): $\log k=s_{f}\left(E_{f}+N_{f}\right)$. Due to ability of the ferrocene moiety to efficiently stabilize the positive charge, ferrocenylphenylmethyl cations constitute a group of very powerful electrofuges $\left(E_{f}>1\right)$. Impact of the phenyl group in ferrocenylphenylmethyl derivatives on stabilization of the positive charge is considerably leveled by the ferrocenyl group, so the rate effect of the alkyl substituents (methyl, ethyl and tert-butyl) on the phenyl ring is suppressed, causing narrow range of $E_{f}$ parameters. Lack of breakdown of Hammett-Brown plot if the rates for the complete set of substrates 1-5 have been correlated, indicates that the ferrocenyl group in $\alpha$-position diminishes the stabilizing effects of electron-donating substituents as well.
\end{abstract}

Keywords: electrofugality, nucleofugalty, ferrocenylphenylmethyl cation, solvolysis, substituent effect.

\section{INTRODUCTION}

$\mathbf{T}$ THE first step in solvolytic $S_{N} 1$ reactions involves the heterolytic cleavage of the carbon-leaving group bond and formation of the carbocation intermediate (electrofuge) and the free leaving group (nucleofuge).[1] The reactivity of a substrate is determined with both abilities the leaving group and the carbocation to depart from the substrate, i.e., with their electrofugality and nucleofugality. A comprehensive electrofugality and nucleofugality scales have been constructed based on solvolytic reactivity of benzhydryl derivatives in various solvents. Accordingly, the heterolysis rate constant of any substrate in a given solvent can be predicted by using the following three-parameter LFER (Equation 1):[2,3]

$$
\log k=s_{f}\left(N_{f}+E_{f}\right)
$$

in which $k$ is first-order rate constant at $25{ }^{\circ} \mathrm{C}, s_{\mathrm{f}}$ is the nucleofuge-specific slope parameter, $N_{f}$ is the nucleofugality in a given solvent, and $E_{\mathrm{f}}$ is the independent variable referring to electrofugality. According to above equation, the nucleofuge specific parameters can be derived from log $k v s . E_{\mathrm{f}}$ plots, ${ }^{[4]}$ while the electrofugalities can be derived from log $k / s_{f}$ vs. $N_{f}$ plots, taking the known $s_{f}$ and $N_{f}$ parameters. The $E_{f}$ values obtained are justified if the linear plots obtained have a slopes of unity. ${ }^{[5]}$

In our previous work we determined the electrofugality of some ferrocenylphenylmethyl cations, mostly those with electron-accepting groups on the phenyl ring $(5$ in Scheme 1). ${ }^{[6]}$ In this work we chose to extend the spectrum of $E_{\mathrm{f}}$ parameters for ferrocenylphenylmethyl substrates by investigating some more reactive ferrocenylphenylmethyl electrofuges (1-4, Scheme 1). The aim was to (a) collect additional $E_{\mathrm{f}}$ parameters that can be used for estimation of the absolute first-order heterolysis rates in a given solvent for variety of substrates that are combination of ferrocenylphenylmethyl moiety and any nucleofuge of known $s_{f}$ and $N_{f}$ parameters, by applying Equation (1), (b) to determine if the stabilizing effect of the ferrocenyl group is similar to that in less reactive substrates, and (c) to make feasible comparison of reactivities of the series of ferrocenylphenylmethyl cations with numerous electrofuges with known $E_{\mathrm{f}}$ values. 

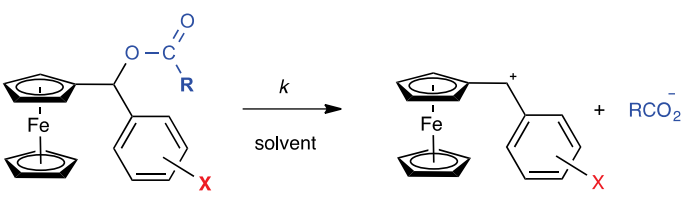

a) $\mathbf{R}=\mathrm{CH}_{3} \mathrm{CH}_{2} \mathrm{CH}_{2}$ (butyrate)

b) $\mathbf{R}=\left(\mathrm{CH}_{3}\right)_{2} \mathrm{CH}$ (isobutyrate)

c) $\mathbf{R}=\mathrm{CH}_{3} \mathrm{CH}_{2} \mathrm{CH}_{2} \mathrm{CH}_{2}$ (valerate)
d) $\mathbf{R}=\left(\mathrm{CH}_{3}\right)_{2} \mathrm{CHCH}_{2}$ (isovalerate)

$$
\begin{aligned}
& \text { 1) } X=4-\mathrm{Me} \\
& \text { 2) } \mathrm{X}=4 \text {-tert-Bu } \\
& \text { 3) } \mathrm{X}=4-\mathrm{Et} \\
& \text { 4) } \mathrm{X}=4-\mathrm{F} \\
& \text { 5) } \mathrm{X}=\mathrm{H}, 4-\mathrm{Br}, 4-\mathrm{Cl} 3-\mathrm{F}, 3-\mathrm{Br}, 3-\mathrm{Cl}
\end{aligned}
$$

Scheme 1. The heterolytic step in the solvolysis of some X-substituted ferrocenylphenylmethyl carboxylates.

To enable measurements using conventional kinetic methods available for us, such a reactive electrofuges should be combined with poor nucleofuges. Up to now the least reactive nucleofuge on the scale is acetate anion ( $N_{\mathrm{f}}$ from -3.55 to -4.8 in various solvents). ${ }^{[6,7]}$ It turned out that acetates of ferrocenylphenylmethyl cations 1-4 solvolyze too fast, so electrofuges $\mathbf{1 - 4}$ should be combined with less reactive nucleofuges. Having in mind that according a qualitative rule of thumb, the abilities of leaving groups are arranged in the same order as the acidities of their conjugate Brönsted acids, we assumed that carboxylates with longer alkyl chain, as are butyrate, isobutyrate, valerate, and isovalerate (a-d, Scheme 1), would be appropriate. Hence, the first step was determining their nucleofugalities.

To get $N_{f}$ and $s_{f}$ values, according to well established procedure, the series of benzhydryl butyrate, isobutyrate, valerate, and isovalerate should have been subjected to kinetic measurement in a given solvent, and the corresponding nucleofuge-specific parameters would have been be derived from log $k$ vs. $E_{\mathrm{f}}$ plots (Equation 1). However, except dianysylmethyl isobutyrate, the synthesis of other substrates failed. Therefore, instead using reactive benzhydryl substrates $\left(E_{f}>0\right)$ to get the nucleofugality parameters from $\log k$ vs. $E_{f}$ plots, we used ferrocenylphenylmethyl derivates whose $E_{\mathrm{f}}$ values have already been detemined. ${ }^{[6]}$

\section{RESULTS AND DISCUSSION}

A series of $X$-substituted ferrocenylphenylmethyl butyrates, isobutyrates, valerates, and isovalerates (5a-5d) which were prepared according to the procedure presented in the Supporting Information, were subjected to solvolysis in various solvents. The solvolysis rates were measured titrimetrically (details are given in Kinetic Methods in the Experimental) at $25{ }^{\circ} \mathrm{C}$ or at least three different temperatures and the rate constants were extrapolated to $25^{\circ} \mathrm{C}$ by using Eyring plot. The first-order rate constants at $25{ }^{\circ} \mathrm{C}$ (measured and extrapolated) are presented in Table $\mathrm{S} 1$ (Supporting Information).

The trends observed from the kinetic data can be summarized as follows. Carboxylates with straight side chains are slightly more reactive than the corresponding carboxylates with branched side chains (Tables S1). Also, the solvolytic reactivities of substrates with non-branched carboxylates (butyrates and valerates) are similar (Figure S1a), whereas those with branched chain somewhat differ, i.e., isovalerate is slightly more reactive that isobutyrate (Figure S1b).

To extract the nucleofugality parameters $\left(N_{\mathrm{f}}\right.$ and $\left.s_{\mathrm{f}}\right)$ for carboxylates $\mathbf{a}-\mathbf{d}$, the logarithms of the first-order solvolysis rate constants in the given solvents were plotted against published $E_{\mathrm{f}}$, values of ferrocenylphenylmethyl cations 5. ${ }^{[6]}$ The correlation lines are presented in Figure S1a and S1b in the Supporting Information. The nucleofugespecific parameters $\left(N_{f}\right.$ and $\left.s_{f}\right)$ are presented in Table 1.

Once the nucleofuge specific parameters for the four carboxylates $\mathbf{a}-\mathbf{d}$ in various solvents have been determined, the electrofugality of ferrocenylphenylmethyl cations 1-4 could be assessed. The substrates prepared were the combination of electrofuges 1-4 and the carboxylates a-d (preparation is presented in Experimental). Solvolysis rates were measured in various aqueous solvents (Table 2 ).

\begin{tabular}{|c|c|c|c|c|c|c|c|c|}
\hline \multirow[t]{2}{*}{ Solvent ${ }^{(a)}$} & \multicolumn{2}{|c|}{ Butyrate } & \multicolumn{2}{|c|}{ Isobutyrate } & \multicolumn{2}{|c|}{ Valerate } & \multicolumn{2}{|c|}{ Isovalerate } \\
\hline & $N_{f}$ & $S f$ & $N_{f}$ & $S f$ & $N_{f}$ & $S f$ & $N_{f}$ & $S f$ \\
\hline $100 \mathrm{M}$ & -4.61 & 0.99 & -4.80 & 1.00 & -4.71 & 0.95 & -4.78 & 0.93 \\
\hline 90M10W & -4.23 & 0.94 & -4.55 & 0.89 & -4.31 & 0.92 & -4.60 & 0.81 \\
\hline 90E10W & -4.58 & 1.03 & -4.70 & 1.03 & -4.61 & 0.97 & -4.68 & 0.93 \\
\hline 80AN20W & -5.15 & 1.01 & -5.24 & 1.04 & -5.28 & 0.97 & -5.38 & 0.95 \\
\hline $80 A 20 W$ & -5.33 & 1.14 & -5.67 & 1.11 & -5.88 & 0.94 & - & - \\
\hline
\end{tabular}

To extract the electrofugalities, $\log k / s_{f}$ vs. $N_{f}$ were plotted for the series of ferrocenylphenylmethyl

Table 1. Nucleofugality parameters $N_{f}$ and $s_{f}$ for butyrate, isobutyrate, valerate and isovalerate in various solvents

\footnotetext{
(a) Binary solvents are given as $v / v ; \mathrm{A}=$ acetone, $\mathrm{AN}=$ acetonitrile, $\mathrm{E}=$ ethanol, $\mathrm{M}=$ methanol, and $\mathrm{W}=$ water.
} 
Table 2. Solvolysis rate constants of some 1-4 ferrocenylphenylmethyl butyrates (a), isobutyrates (b), valerates (c), and isovalerates (d) in various solvents at $25^{\circ} \mathrm{C}$

\begin{tabular}{|c|c|c|c|c|}
\hline Substrate & Electrofuge $^{(a)}$ & $L G^{(b)}$ & Solvent ${ }^{(c)}$ & $k / s^{-1(d)}$ \\
\hline $1 a$ & 4- $\mathrm{MeC}_{6} \mathrm{H}_{5} \mathrm{FcCH}^{+}$ & But & 80AN20W & $4.23 \times 10^{-3(e, f)}$ \\
\hline \multirow[t]{2}{*}{$1 b$} & & Isobut & 90E10W & $1.09 \times 10^{-2(e, g)}$ \\
\hline & & & 80AN20W & $3.02 \pm 0.03) \times 10^{-3}$ \\
\hline $1 \mathrm{c}$ & & Val & 80AN2OW & $3.97 \times 10^{-3(e, h)}$ \\
\hline $1 d$ & & Isoval & 80AN20W & $(3.66 \pm 0.06) \times 10^{-3}$ \\
\hline $2 a$ & 4-tButC ${ }_{6} \mathrm{H}_{5} \mathrm{FcCH}^{+}$ & But & 80AN20W & $(3.62 \pm 0.04) \times 10^{-3}$ \\
\hline $2 b$ & & Isobut & 90E10W & $1.06 \times 10^{-2(e, i)}$ \\
\hline $2 c$ & & Val & 80AN20W & $(3.46 \pm 0.02) \times 10^{-3}$ \\
\hline $2 d$ & & Isoval & 80AN20W & $(3.21 \pm 0.06) \times 10^{-4}$ \\
\hline $3 a$ & $4-\mathrm{EtC}_{6} \mathrm{H}_{5} \mathrm{FcCH}^{+}$ & But & 80AN20W & $(3.73 \pm 0.05) \times 10^{-3}$ \\
\hline \multirow[t]{2}{*}{$3 b$} & & Isobut & 90E10W & $8.47 \times 10^{-3(e, j)}$ \\
\hline & & & 80AN20W & $(2.31 \pm 0.03) \times 10^{-3}$ \\
\hline $3 c$ & & Val & 80AN2OW & $(2.91 \pm 0.01) \times 10^{-3}$ \\
\hline \multirow[t]{2}{*}{$4 a$} & 4- $\mathrm{FC}_{6} \mathrm{H}_{5} \mathrm{FcCH}^{+}$ & But & 90E10W & $4.75 \times 10^{-3(e, k)}$ \\
\hline & & & 80AN2OW & $(1.45 \pm 0.03) \times 10^{-3}$ \\
\hline \multirow[t]{2}{*}{$4 b$} & & Isobut & 90E10W & $(3.65 \pm 0.03) \times 10^{-3}$ \\
\hline & & & 80AN20W & $(1.05 \pm 0.01) \times 10^{-3}$ \\
\hline \multirow[t]{2}{*}{$4 c$} & & Val & 80AN2OW & $(1.22 \pm 0.07) \times 10^{-3}$ \\
\hline & & Isoval & 80AN2OW & $(1.32 \pm 0.01) \times 10^{-3}$ \\
\hline
\end{tabular}

(a) $\mathrm{FC}=$ ferrocene.

(b) But $=\mathrm{CH}_{3} \mathrm{CH}_{2} \mathrm{CH}_{2} \mathrm{COO}^{-}$, Isobut $=\left(\mathrm{CH}_{3}\right)_{2} \mathrm{CHCOO}^{-}$, Val $=\mathrm{CH}_{3} \mathrm{CH}_{2} \mathrm{CH}_{2} \mathrm{CH}_{2} \mathrm{COO}^{-}$, and Isoval $=\left(\mathrm{CH}_{3}\right)_{2} \mathrm{CHCH}_{2} \mathrm{COO}^{-}$.

(c) Binary solvents are given as $v / v$ at $25^{\circ} \mathrm{C}$; $\mathrm{AN}=$ acetonitrile, $\mathrm{E}=$ ethanol, $\mathrm{W}=$ water.

(d) Average rate constants from at least three runs performed at $25^{\circ} \mathrm{C}$. Errors are SD.

(e) Extrapolated from data at different temperatures by use of the Eyring equation; errors for activation parameters shown are standard errors.

(f) $\Delta H^{\ddagger}=66.0 \pm 0.9 \mathrm{kJmol}^{-1}, \Delta S^{\ddagger}=-68.9 \pm 3.0 \mathrm{JK}^{-1} \mathrm{~mol}^{-1}$.

(g) $\Delta H^{\ddagger}=71.3 \pm 0.1 \mathrm{kJmol}^{-1}, \Delta S^{\ddagger}=-43.3 \pm 0.5 \mathrm{JK}^{-1} \mathrm{~mol}^{-1}$.

(h) $\Delta H^{\ddagger}=69.5 \pm 0.8 \mathrm{kJmol}^{-1}, \Delta S^{\ddagger}=-58.0 \pm 2.9 \mathrm{JK}^{-1} \mathrm{~mol}^{-1}$.

(i) $\Delta H^{\ddagger}=73.3 \pm 1.1 \mathrm{kJmol}^{-1}, \Delta S^{\ddagger}=-36.9 \pm 3.8 \mathrm{JK}^{-1} \mathrm{~mol}^{-1}$.

(j) $\Delta H^{\ddagger}=61.9 \pm 3.2 \mathrm{kJmol}^{-1}, \Delta S^{\ddagger}=-76.8 \pm 11.6 \mathrm{JK}^{-1} \mathrm{~mol}^{-1}$.

(k) $\Delta H^{\ddagger}=51.3 \pm 1.5 \mathrm{kJmol}^{-1}, \Delta S^{\ddagger}=-117.4 \pm 5.1 \mathrm{JK}^{-1} \mathrm{~mol}^{-1}$.

carboxylates 1-4 (Figure 1). The weighted average of the slopes of correlation lines in Figure 1 is $0.9969 \pm 0.004$. The electrofugalities obtained from the correlations are shown in Table 3 in which, for sake of comparison, the electrofugality of some less reactive ferrocenylphenylmethyl cations as well as benzhydryl cations are presented.

By comparing the $E_{f}$ values of ferrocenylphenylmethyl cations and benzhydryl cations it is obvious that the effect of the substituent on the phenyl ring is much more pronounced in benzhydryl derivatives. Thus, switching from 4-methyl to 3-chloro substituent in benzhydryl substrates cause decrease of $E_{\mathrm{f}}$ values for about three units, which roughly corresponds to difference in reactivity of three orders of magnitude. On the other hand, the difference of $E_{f}$ parameters between 3-chloroferrocenylphenylmethyl cation and 4-methylferrocenylphenylmethyl cation is about one unit, i.e., 4-methyl derivative is only about ten times more reactive than 3-chloro derivative.

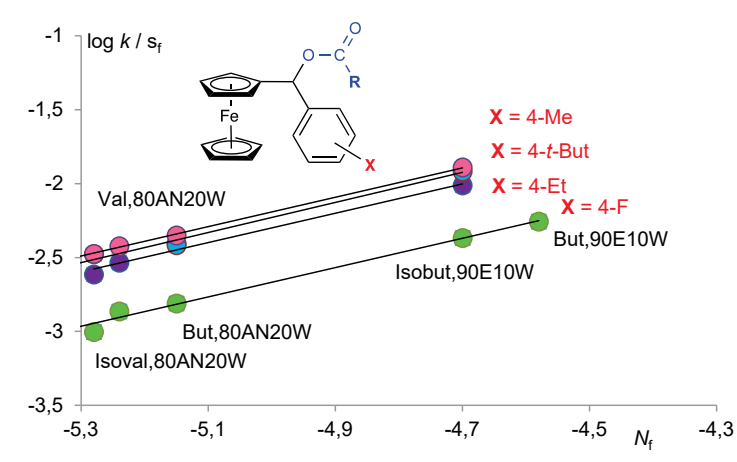

Figure 1. Plots $\log k / s_{f}$ against $N_{f}$ for the solvolysis of substituted ferrocenylphenylmethyl butyrates, isobutyrates, valerates, and isovalerates 1-4 in various solvents. But $=\mathrm{CH}_{3} \mathrm{CH}_{2} \mathrm{CH}_{2} \mathrm{COO}^{-}$ Isobut $=\left(\mathrm{CH}_{3}\right)_{2} \mathrm{CHCOO}^{-}$, Val $=\mathrm{CH}_{3} \mathrm{CH}_{2} \mathrm{CH}_{2} \mathrm{CH}_{2} \mathrm{COO}^{-}$, Isoval = $\left(\mathrm{CH}_{3}\right)_{2} \mathrm{CHCH}_{2} \mathrm{COO}$. Binary solvents are given as $\mathrm{V} / \mathrm{V}$. $\mathrm{AN}=$ acetonitrile, $\mathrm{E}=$ ethanol, and $\mathrm{W}=$ water. 
Table 3. Electrofugality parameters of some substituted ferrocenylphenylmethyl cations and the corresponding benzhydryl cations.

\begin{tabular}{ccc}
\hline \multirow{2}{*}{ Substituent X } & \multicolumn{2}{c}{$E_{f}$} \\
\cline { 2 - 3 } & $\begin{array}{c}\text { Ferrocenyl-X- } \\
\text { phenylmethyl cation }\end{array}$ & $\begin{array}{c}\text { X-Benzhydryl } \\
\text { cation }^{(\text {b) }}\end{array}$ \\
\hline 4-Me (1) & 2.80 & -4.63 \\
4-tBut (2) & 2.83 & \\
4-Et (3) & 2.68 & -6.03 \\
$\mathrm{H}$ & $2.40^{(\mathrm{a})}$ & -5.72 \\
$4-\mathrm{F}(4)$ & 2.31 & -6.62 \\
$4-\mathrm{Br}$ & $2.15^{(\mathrm{a})}$ & -7.53 \\
$3-\mathrm{F}$ & $1.77^{(\mathrm{a})}$ & -7.69 \\
$3-\mathrm{Cl}$ & $1.67^{(\mathrm{a})}$ & \\
\hline
\end{tabular}

(a) $E_{\mathrm{f}}$ parameters for ferrocenyl-X-phenylmethyl cations $(\mathrm{X}=\mathrm{H}, 4-\mathrm{Br}, 3-\mathrm{F}$, 3-Cl) are taken from Ref. [6].

(b) $E_{\mathrm{f}}$ parameters for benzhydryl cations are taken from Ref. [3a].

Kinetic results obtained earlier with the series of ferrocenylphenylmethyl derivatives $\mathbf{5}$ as well as quantum chemical calculations showed that the effect of the substituents on the phenyl ring is suppressed due to very strong electron donating ability of the $\alpha$-ferrocenyl group, i.e., leveling occurs. ${ }^{[6]}$ The question arose if this applied to all $X$ substituents (1-5), or electron-donating substituents on the phenyl in substrates 1-3 had somewhat more pronounced effect than in $\mathbf{5}$. This assumption was tested with Hammett-Brown correlation. If the effects of electrondonating substituents in 1-3 were stronger than those in the series of $\mathbf{5}$ examined earlier, breakdown of the $\sigma^{+}, \rho^{+}$ correlation line would occur. However, as presented in Figure 2, linear correlation has been obtained if all data for substrates 1-5 are included. In the limits of experimental error, essentially the same slope has been obtained in e.g. $90 \%$ aq. ethanol for the complete set of ferrocenylphenylmethyl isoburtyrates $\mathbf{1 - 5}\left(\rho^{+}=-1.60\right)$ and that for the series of 5 determined earlier $\left(\rho^{+}=-1.46\right)$, due to very strong electron-donating ability of the ferrocenyl group (Figure 2).

\section{EXPERIMENTAL}

Substrate Preparation: The ferrocenyl phenyl ketones were prepared by standard acylation. ${ }^{[8]}$ using the corresponding benzoyl chloride and ferrocene in dichloromethane with $\mathrm{AlCl}_{3}$ as catalyst at low $\left(0^{\circ} \mathrm{C}\right)$ or ambient temperature. Reduction of ferrocenyl phenyl ketones with $\mathrm{LiAlH}_{4}$ in diethyl ether according to published procedure ${ }^{[9]}$ gave 4-fluorophenylferrocenemethanol (69\%), 4-methylphenylferrocenemethanol (86\%), 4-ethylphenylferrocenemethanol (77\%), and 4-tert-butylphenylferrocenemethanol (66\%), respectively.

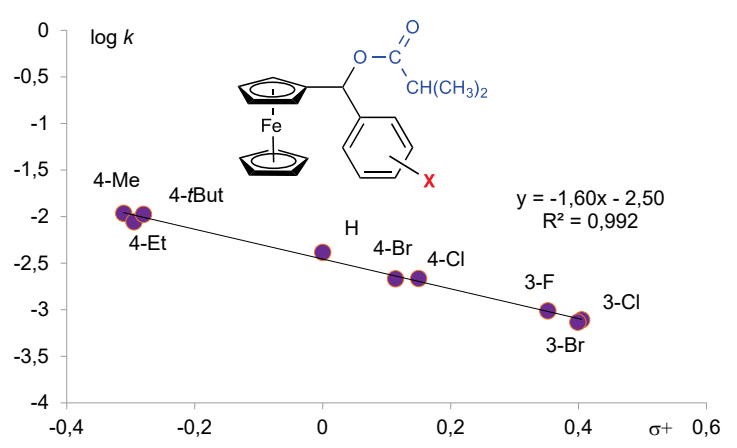

Figure 2. Hammett-Brown plot for the solvolysis of $X$-substituted ferrocenylphenylmethyl isobutyrates in $90 \%$ aq. ethanol at $25^{\circ} \mathrm{C}$.

General Procedure for the Synthesis of Ferrocenylphenylmethyl Butyrates, Isobutyrates, Valerates, and Isovalerates: A solution of butyryl, isobutyryl, valeroyl, and isovaleryl chloride $(\approx 2 \mathrm{eq})$ in anhydrous benzene $(10 \mathrm{~mL}$ ) was added dropwise to a previously prepared stirred solution of the appropiate phenylferrocenemethanol (1 eq) and pyridine $(0.5 \mathrm{~g}, 6.32 \mathrm{mmol})$ in anhydrous benzene (10 $\mathrm{mL}$ ). The reaction mixture was stirred under argon at ambient temperature for from $1-2 \mathrm{~h}$ to overnight Precipitated pyridinium chloride was removed by filtration, and excess of pyridine was removed with hydrochloride acid (15 mL, $5 \%$ ). The benzene layer was separated and washed with concentrated solution of sodium hydroxide and water. After drying over anhydrous sodium sulfate, benzene was evaporated in vacuum. Butyrates, isobutyrates, valerates, and isovalerates were obtained as dark red crystals or oils (yield 44.2-85.9\%).

4-Methylphenylferrocenylmethyl Butyrate (1a): This compound was obtained from 4-methylphenylferrocenemethanol (0.5 g, $1.63 \mathrm{mmol})$, pyridine $(0.5 \mathrm{~g}, 6.32 \mathrm{mmol})$, and butyryl chloride $(0.35 \mathrm{~g}, 3.28 \mathrm{~mol})$; yield $0.46 \mathrm{~g}, 74.8 \%$. ${ }^{1} \mathrm{H} \mathrm{NMR}\left(400 \mathrm{MHz}, \mathrm{CDCl}_{3}, 25^{\circ} \mathrm{C}\right): \delta / \mathrm{ppm}=0.93(\mathrm{t}, J=7.4 \mathrm{~Hz}$, $\left.3 \mathrm{H} ; \mathrm{CH}_{3} \mathrm{CH}_{2} \mathrm{CH}_{2} \mathrm{COOR}\right)$, 1.64-1.70 (m, 5H; $\mathrm{CH}_{3} \mathrm{CH}_{2} \mathrm{CH}_{2} \mathrm{COOR}$ $+\mathrm{CH}_{3} \mathrm{Ar}$ ), $2.34\left(\mathrm{t}, \mathrm{J}=7.4 \mathrm{~Hz}, 2 \mathrm{H} ; \mathrm{CH}_{3} \mathrm{CH}_{2} \mathrm{CH}_{2} \mathrm{COOR}\right), 4.0-4.30$ $\left(\mathrm{m}, 4 \mathrm{H} ; \mathrm{C}_{5} \mathrm{H}_{4}\right), 4.09\left(\mathrm{~s}, 5 \mathrm{H} ; \mathrm{C}_{5} \mathrm{H}_{5}\right), 6.72(\mathrm{~s}, 1 \mathrm{H} ; \mathrm{ArCHFc}), 7.13$ (d, J = 7.9 Hz, 2H; Ar), 7.29 (d, J = 8.0 Hz, $2 \mathrm{H} ; \mathrm{Ar}) .{ }^{13} \mathrm{C} \mathrm{NMR}$ (100 MHz, CDCl $3,25^{\circ} \mathrm{C}$ ): $\delta / \mathrm{ppm}=13.8\left(\mathrm{CH}_{3} \mathrm{CH}_{2} \mathrm{CH}_{2} \mathrm{COOR}\right)$, $18.6\left(\mathrm{CH}_{3} \mathrm{CH}_{2} \mathrm{CH}_{2} \mathrm{COOR}\right), 21.3\left(\mathrm{CH}_{3} \mathrm{Ar}\right), 36.6\left(\mathrm{CH}_{3} \mathrm{CH}_{2} \mathrm{CH}_{2} \mathrm{COOR}\right)$, 67.5, 67.7, 68.2, 68.3 $\left(\mathrm{C}_{5} \mathrm{H}_{4}\right), 69.0\left(\mathrm{C}_{5} \mathrm{H}_{5}\right), 74.0$ (ArCHFc), 88.4 (Fc), 127.2, 129.0, 137.5, 137.7 (Ar), 172.7 (COOR).

4-tert-Butylphenylferrocenylmethyl Butyrate (2a): This compound was obtained from 4-tert-butylphenylferrocenemethanol (0.5 g, $1.44 \mathrm{mmol})$, pyridine $(0.5 \mathrm{~g}, 6.32$ $\mathrm{mmol})$, and butyryl chloride $(0.30 \mathrm{~g}, 2.82 \mathrm{~mol})$; yield $0.42 \mathrm{~g}$, $69.4 \% .{ }^{1} \mathrm{H}$ NMR $\left(400 \mathrm{MHz}, \mathrm{CDCl}_{3}, 25{ }^{\circ} \mathrm{C}\right): \delta / \mathrm{ppm}=0.95$ (t, J = 7.3 Hz, 3H; $\left.\mathrm{CH}_{3} \mathrm{CH}_{2} \mathrm{CH}_{2} \mathrm{COOR}\right), 1.31\left(\mathrm{~s}, 9 \mathrm{H},\left(\mathrm{CH}_{3}\right)_{3} \mathrm{CAr}\right)$, 1.67-1.72(m, 2H; $\left.\mathrm{CH}_{3} \mathrm{CH}_{2} \mathrm{CH}_{2} \mathrm{COOR}\right), 2.35(\mathrm{t}, J=7.3 \mathrm{~Hz}, 2 \mathrm{H}$; 
$\mathrm{CH}_{3} \mathrm{CH}_{2} \mathrm{CH}_{2} \mathrm{COOR}$ ), 4.04-4.31 (m, 4H; $\left.\mathrm{C}_{5} \mathrm{H}_{4}\right), 4.10(\mathrm{~s}, 5 \mathrm{H}$; $\left.\mathrm{C}_{5} \mathrm{H}_{5}\right), 6.74(\mathrm{~s}, 1 \mathrm{H} ; \mathrm{ArCHFc}), 7.29-7.39(\mathrm{~m}, 4 \mathrm{H} ; \mathrm{Ar}) .{ }^{13} \mathrm{C} \mathrm{NMR}$ $\left(100 \mathrm{MHz}, \mathrm{CDCl}_{3}, 25^{\circ} \mathrm{C}\right): \delta / \mathrm{ppm}=13.8\left(\mathrm{CH}_{3} \mathrm{CH}_{2} \mathrm{CH}_{2} \mathrm{COOR}\right)$, $18.5\left(\mathrm{CH}_{3} \mathrm{CH}_{2} \mathrm{CH}_{2} \mathrm{COOR}\right), 31.6\left(\left(\mathrm{CH}_{3}\right)_{3} \mathrm{CAr}\right), 34.6\left(\left(\mathrm{CH}_{3}\right)_{3} \mathrm{CAr}\right)$, $36.6\left(\mathrm{CH}_{3} \mathrm{CH}_{2} \mathrm{CH}_{2} \mathrm{COOR}\right), 67.5,67.5,68.0,68.1\left(\mathrm{C}_{5} \mathrm{H}_{4}\right), 68.8$ $\left(\mathrm{C}_{5} \mathrm{H}_{5}\right), 73.7$ ( $\left.\mathrm{ArCHFC}\right), 88.4(\mathrm{Fc}), 125.1,126.8,137.4,150.7$ (Ar), 172.7 (COOR).

4-Ethylphenylferrocenylmethyl Butyrate (3a): This compound was obtained from 4-ethylphenylferrocenemethanol $(0.5 \mathrm{~g}, 1.56 \mathrm{mmol})$, pyridine $(0.5 \mathrm{~g}, 6.32 \mathrm{mmol})$, and butyryl chloride $(0.30 \mathrm{~g}, 2.82 \mathrm{~mol})$; yield $0.46 \mathrm{~g}, 75.6 \%$. ${ }^{1 \mathrm{H}} \mathrm{NMR}$ $\left(400 \mathrm{MHz}, \mathrm{CDCl}_{3}, 25^{\circ} \mathrm{C}\right): \delta / \mathrm{ppm}=0.94(\mathrm{t}, J=7.4 \mathrm{~Hz}, 3 \mathrm{H}$; $\mathrm{CH}_{3} \mathrm{CH}_{2} \mathrm{CH}_{2} \mathrm{COOR}$ ), 1.23 (t, J=7.6 Hz, 3H; $\mathrm{CH}_{3} \mathrm{CH}_{2} \mathrm{Ar}$ ), 1.65$1.71\left(\mathrm{~m}, 2 \mathrm{H} ; \mathrm{CH}_{3} \mathrm{CH}_{2} \mathrm{CH}_{2} \mathrm{COOR}\right), 2.34(\mathrm{t}, J=7.4 \mathrm{~Hz}, 2 \mathrm{H}$; $\mathrm{CH}_{3} \mathrm{CH}_{2} \mathrm{CH}_{2} \mathrm{COOR}$ ), 2.64 (q, J= $7.6 \mathrm{~Hz}, 2 \mathrm{H} ; \mathrm{CH}_{3} \mathrm{CH}_{2} \mathrm{Ar}$ ), 4.02$4.31\left(\mathrm{~m}, 4 \mathrm{H} ; \mathrm{C}_{5} \mathrm{H}_{4}\right), 4.10\left(\mathrm{~s}, 5 \mathrm{H} ; \mathrm{C}_{5} \mathrm{H}_{5}\right), 6.73(\mathrm{~s}, 1 \mathrm{H} ; \mathrm{ArCHFc})$, $7.16(\mathrm{~d}, J=7.9 \mathrm{~Hz}, 2 \mathrm{H} ; \mathrm{Ar}) .7 .31(\mathrm{~d}, J=8.1 \mathrm{~Hz}, 2 \mathrm{H} ; \mathrm{Ar}) .{ }^{13} \mathrm{C} \mathrm{NMR}$ $\left(100 \mathrm{MHz}, \mathrm{CDCl}_{3}, 25^{\circ} \mathrm{C}\right): \delta / \mathrm{ppm}=13.9\left(\mathrm{CH}_{3} \mathrm{CH}_{2} \mathrm{CH}_{2} \mathrm{COOR}\right)$, $15.6\left(\mathrm{CH}_{3} \mathrm{CH}_{2} \mathrm{Ar}\right), 18.6\left(\mathrm{CH}_{3} \mathrm{CH}_{2} \mathrm{CH}_{2} \mathrm{COOR}\right), 28.7\left(\mathrm{CH}_{3} \mathrm{CH}_{2} \mathrm{Ar}\right)$, $36.7\left(\mathrm{CH}_{3} \mathrm{CH}_{2} \mathrm{CH}_{2} \mathrm{COOR}\right), 67.6,67.7,68.2,68.3\left(\mathrm{C}_{5} \mathrm{H}_{4}\right), 69.0$ $\left(\mathrm{C}_{5} \mathrm{H}_{5}\right), 74.0$ (ArCHFc), 88.5 (Fc), 127.3, 127.8, 137.7, 144.0 (Ar), 172.8 (COOR).

4-Fluorophenylferrocenylmethyl Butyrate (4a): This compound was obtained from 4-fluorophenylferrocenemethanol $(0.5 \mathrm{~g}, 1.61 \mathrm{mmol})$, pyridine $(0.5 \mathrm{~g}, 6.32 \mathrm{mmol})$, and butyryl chloride $(0.27 \mathrm{~g}, 2.53 \mathrm{mmol})$; yield $0.38 \mathrm{~g}, 62.1 \%$. ${ }^{1} \mathrm{H} \mathrm{NMR}\left(400 \mathrm{MHz}, \mathrm{CDCl}_{3}, 25^{\circ} \mathrm{C}\right) \delta / \mathrm{ppm}=0.93(\mathrm{t}, J=7.4 \mathrm{~Hz}$, $\left.3 \mathrm{H} ; \mathrm{CH}_{3} \mathrm{CH}_{2} \mathrm{CH}_{2} \mathrm{COOR}\right), 1.65-1.71\left(\mathrm{~m}, 2 \mathrm{H} ; \mathrm{CH}_{3} \mathrm{CH}_{2} \mathrm{CH}_{2} \mathrm{COOR}\right)$, $2.34\left(\mathrm{t}, J=7.5 \mathrm{~Hz}, 2 \mathrm{H} ; \mathrm{CH}_{3} \mathrm{CH}_{2} \mathrm{CH}_{2} \mathrm{COOR}\right.$ ), 3.98-4.30 (m, $\left.4 \mathrm{H} ; \mathrm{C}_{5} \mathrm{H}_{4}\right), 4.10\left(\mathrm{~s}, 5 \mathrm{H} ; \mathrm{C}_{5} \mathrm{H}_{5}\right), 6.73(\mathrm{~s}, 1 \mathrm{H} ; \operatorname{ArCHFc})$, 7.00-7.40 (m, 4H; Ar). ${ }^{19} \mathrm{~F}$ NMR (376 MHz, $\left.\mathrm{CDCl}_{3}, 25^{\circ} \mathrm{C}\right)$ : $\delta / \mathrm{ppm}=-114.06(\mathrm{~m}) .{ }^{13} \mathrm{C} \mathrm{NMR}\left(100 \mathrm{MHz}, \mathrm{CDCl}_{3}, 25^{\circ} \mathrm{C}\right):$ $\delta / p p m=13.8\left(\mathrm{CH}_{3} \mathrm{CH}_{2} \mathrm{CH}_{2} \mathrm{COOR}\right), 18.6\left(\mathrm{CH}_{3} \mathrm{CH}_{2} \mathrm{CH}_{2} \mathrm{COOR}\right)$, $36.6\left(\mathrm{CH}_{3} \mathrm{CH}_{2} \mathrm{CH}_{2} \mathrm{COOR}\right), 67.4,67.6,68.3,68.4\left(\mathrm{C}_{5} \mathrm{H}_{4}\right), 69.0$ $\left(\mathrm{C}_{5} \mathrm{H}_{5}\right), 73.5$ ( $\left.\mathrm{ArCHFc}\right), 88.0(\mathrm{Fc}), 115.2$ (d, $\left.J=21.5 \mathrm{~Hz} ; \mathrm{Ar}-\mathrm{F}\right)$, 129.1 (d, J = 8.1 Hz; Ar-F), 136.4 (d, J = 3.3 Hz; Ar-F), 162.4 (d, $J=246.5 \mathrm{~Hz} ; \mathrm{Ar}-\mathrm{F}), 172.7$ (COOR).

4-Methylphenylferrocenylmethyl Isobutyrate (1b): This compound was obtained from 4-methylphenylferrocenemethanol $(0.5 \mathrm{~g}, 1.63 \mathrm{mmol})$, pyridine $(0.5 \mathrm{~g}, 6.32 \mathrm{mmol})$, and isobutyryl chloride $(0.35 \mathrm{~g}, 3.28 \mathrm{~mol})$; yield $0.42 \mathrm{~g}$, $68.7 \% .{ }^{1} \mathrm{H} \mathrm{NMR}\left(400 \mathrm{MHz}, \mathrm{CDCl}_{3}, 25^{\circ} \mathrm{C}\right): \delta / \mathrm{ppm}=1.18-1.22$ $\left(\mathrm{d}+\mathrm{d}, J=7.0 \mathrm{~Hz}+J=7.0 \mathrm{~Hz}, 3 \mathrm{H}+3 \mathrm{H} ;\left(\mathrm{CH}_{3}\right)_{2} \mathrm{CHCOOR}\right), 2.33$ (s, $\left.3 \mathrm{H} ; \mathrm{CH}_{3} \mathrm{Ar}\right)$, 2.57-2.64 (m, $\left.1 \mathrm{H} ;\left(\mathrm{CH}_{3}\right)_{2} \mathrm{CHCOOR}\right), 4.00-4.29$ $\left(\mathrm{m}, 4 \mathrm{H} ; \mathrm{C}_{5} \mathrm{H}_{4}\right), 4.11\left(\mathrm{~s}, 5 \mathrm{H} ; \mathrm{C}_{5} \mathrm{H}_{5}\right), 6.70(\mathrm{~s}, 1 \mathrm{H} ; \mathrm{ArCHFc}), 7.13(\mathrm{~d}, J$ $=8.0 \mathrm{~Hz}, 2 \mathrm{H} ; \mathrm{Ar}), 7.27$ (d, J=8.0 Hz, 2H; Ar). ${ }^{13} \mathrm{C} \mathrm{NMR}(100 \mathrm{MHz}$, $\left.\mathrm{CDCl}_{3}, 25^{\circ} \mathrm{C}\right): \delta / \mathrm{ppm}=19.0+19.0\left(\left(\mathrm{CH}_{3}\right)_{2} \mathrm{CHCOOR}\right), 21.2$ $\left(\mathrm{CH}_{3} \mathrm{Ar}\right), 34.2\left(\left(\mathrm{CH}_{3}\right)_{2} \mathrm{CHCOOR}\right), 67.3,67.3,68.0,68.1\left(\mathrm{C}_{5} \mathrm{H}_{4}\right)$, $68.8\left(\mathrm{C}_{5} \mathrm{H}_{5}\right), 73.9$ (ArCHFc), 88.5 (Fc), 127.0, 129.0, 137.6, 137.6 (Ar), 176.0 (COOR). 4-tert-butylphenylferrocenylmethyl Isobutyrate (2b): This compound was obtained from 4-tert-butylphenylferrocenemethanol $(0.5 \mathrm{~g}, 1.44 \mathrm{mmol})$, pyridine $(0.5 \mathrm{~g}, 6.32$ $\mathrm{mmol})$, and isobutyryl chloride $(0.21 \mathrm{~g}, 1.97 \mathrm{~mol})$; yield 0.43 g, $71.5 \% .{ }^{1} \mathrm{H} \mathrm{NMR}\left(400 \mathrm{MHz}, \mathrm{CDCl}_{3}, 25^{\circ} \mathrm{C}\right): \delta / \mathrm{ppm}=1.21-$ $1.25\left(\mathrm{~d}+\mathrm{d}, J=7.0 \mathrm{~Hz}+J=7.0 \mathrm{~Hz}, 3 \mathrm{H}+3 \mathrm{H} ;\left(\mathrm{CH}_{3}\right)_{2} \mathrm{CHCOOR}\right)$, $\left.1.31\left(\mathrm{~s}, 9 \mathrm{H} ;\left(\mathrm{CH}_{3}\right)_{3} \mathrm{CAr}\right)\right), 2.60-2.67\left(\mathrm{~m}, 1 \mathrm{H} ;\left(\mathrm{CH}_{3}\right)_{2} \mathrm{CHCOOR}\right)$, 4.04-4.31 (m, 4H; $\left.\mathrm{C}_{5} \mathrm{H}_{4}\right), 4.12\left(\mathrm{~s}, 5 \mathrm{H} ; \mathrm{C}_{5} \mathrm{H}_{5}\right), 6.73(\mathrm{~s}, 1 \mathrm{H}$; ArCHFc), 7.29-7.36 (m, 4H; Ar). ${ }^{13} \mathrm{C} \mathrm{NMR} \mathrm{(100} \mathrm{MHz,} \mathrm{CDCl}_{3}$, $\left.25^{\circ} \mathrm{C}\right): \delta / \mathrm{ppm}=19.1+19.2\left(\left(\mathrm{CH}_{3}\right)_{2} \mathrm{CHCOOR}\right), 31.5\left(\left(\mathrm{CH}_{3}\right)_{3} \mathrm{CAr}\right)$, $34.4\left(\left(\mathrm{CH}_{3}\right)_{2} \mathrm{CHCOOR}\right), 34.6\left(\left(\mathrm{CH}_{3}\right)_{3} \mathrm{CAr}\right), 67.3,67.4,68.1$, $68.1\left(\mathrm{C}_{5} \mathrm{H}_{4}\right), 69.0\left(\mathrm{C}_{5} \mathrm{H}_{5}\right), 73.8(\mathrm{ArCHFC}), 88.8(\mathrm{Fc}), 125.2$, $126.8,137.7,150.8$ (Ar), 176.1 (COOR).

4-Ethylphenylferrocenylmethyl Isobutyrate (3b): This compound was obtained from 4-ethylphenylferrocenemethanol $(0.5 \mathrm{~g}, 1.56 \mathrm{mmol})$, pyridine $(0.5 \mathrm{~g}, 6.32 \mathrm{mmol})$, and butyryl chloride $(0.27 \mathrm{~g}, 2.53 \mathrm{~mol})$; yield $0.42 \mathrm{~g}, 69.2 \%$. ${ }^{1} \mathrm{H} \mathrm{NMR}\left(400 \mathrm{MHz}, \mathrm{CDCl}_{3}, 25^{\circ} \mathrm{C}\right): \delta / \mathrm{ppm}=1.18-1.24(\mathrm{~m}, 9 \mathrm{H}$; $\left.\left(\mathrm{CH}_{3}\right)_{2} \mathrm{CHCOOR}+\mathrm{CH}_{3} \mathrm{CH}_{2} \mathrm{Ar}\right), 2.60-2.66\left(\mathrm{~m}, 3 \mathrm{H} ;\left(\mathrm{CH}_{3}\right)_{2} \mathrm{CHCOOR}\right.$ $\left.+\mathrm{CH}_{3} \mathrm{CH}_{2} \mathrm{Ar}\right), 4.01-4.29\left(\mathrm{~m}, 4 \mathrm{H} ; \mathrm{C}_{5} \mathrm{H}_{4}\right), 4.11\left(\mathrm{~s}, 5 \mathrm{H} ; \mathrm{C}_{5} \mathrm{H}_{5}\right)$, 6.71 (s, 1H; $\operatorname{ArCHFc}), 7.16$ (d, J = 7.9 Hz, 2H; Ar), 7.30 (d, $J=$ $8.0 \mathrm{~Hz}, 2 \mathrm{H} ; \mathrm{Ar}) .{ }^{13} \mathrm{C} \mathrm{NMR}\left(100 \mathrm{MHz}, \mathrm{CDCl}_{3}, 25^{\circ} \mathrm{C}\right): \delta / \mathrm{ppm}=$ $15.6\left(\mathrm{CH}_{3} \mathrm{CH}_{2} \mathrm{Ar}\right), \quad 19.1+19.2\left(\left(\mathrm{CH}_{3}\right)_{2} \mathrm{CHCOOR}\right), 28.7$ $\left(\mathrm{CH}_{3} \mathrm{CH}_{2} \mathrm{Ar}\right), 34.4\left(\left(\mathrm{CH}_{3}\right)_{2} \mathrm{CHCOOR}\right), 67.4,67.4,68.1,68.2$ $\left(\mathrm{C}_{5} \mathrm{H}_{4}\right), 69.0\left(\mathrm{C}_{5} \mathrm{H}_{5}\right), 73.9$ (ArCHFc), $88.7(\mathrm{Fc}), 127.1,127.8$, 137.3, 144.0 (Ar), 176.1 (COOR).

4-Fluorophenylferrocenylmethyl Isobutyrate (4b): This compound was obtained from 4-fluorophenylferrocenemethanol (0.5 g, $1.61 \mathrm{mmol})$, pyridine $(0.5 \mathrm{~g}, 6.32 \mathrm{mmol})$, and butyryl chloride $(0.21 \mathrm{~g}, 1.97 \mathrm{mmol})$; yield $0.43 \mathrm{~g}, 70.2 \%$. ${ }^{1} \mathrm{H}$ NMR $\left(400 \mathrm{MHz} \mathrm{CDCl}_{3}, 25^{\circ} \mathrm{C}\right): \delta / \mathrm{ppm}=1.18-1.22(\mathrm{~d}+\mathrm{d}$, $\left.J=7.0 \mathrm{~Hz}+J=7.0 \mathrm{~Hz}, 3 \mathrm{H}+3 \mathrm{H} ;\left(\mathrm{CH}_{3}\right)_{2} \mathrm{CHCOOR}\right), 2.58-2.65$ (m, $\left.1 \mathrm{H} ;\left(\mathrm{CH}_{3}\right)_{2} \mathrm{CHCOOR}\right), 3.98-4.29\left(\mathrm{~m}, 4 \mathrm{H} ; \mathrm{C}_{5} \mathrm{H}_{4}\right), 4.11(\mathrm{~s}$, $\left.5 \mathrm{H} ; \mathrm{C}_{5} \mathrm{H}_{5}\right), 6.71(\mathrm{~s}, 1 \mathrm{H} ; \mathrm{ArCHFc}), 7.00-7.38(\mathrm{~m}, 4 \mathrm{H} ; \mathrm{Ar}) .{ }^{19} \mathrm{~F}$ NMR (376 MHz, $\left.\mathrm{CDCl}_{3}, 25^{\circ} \mathrm{C}\right): \delta / \mathrm{ppm}=-114.1(\mathrm{~m}) .{ }^{13} \mathrm{C} \mathrm{NMR}$ $\left(100 \mathrm{MHz}, \mathrm{CDCl}_{3}, 25^{\circ} \mathrm{C}\right): \delta / \mathrm{ppm}=19.1+19.1\left(\left(\mathrm{CH}_{3}\right)_{2} \mathrm{CHCOOR}\right)$, $34.3\left(\left(\mathrm{CH}_{3}\right)_{2} \mathrm{CHCOOR}\right), 67.3,67.34,68.3,68.3\left(\mathrm{C}_{5} \mathrm{H}_{4}\right), 69.0$ $\left(\mathrm{C}_{5} \mathrm{H}_{5}\right), 73.4$ ( $\left.\mathrm{ArCHFc}\right), 88.2(\mathrm{Fc}), 115.3$ (d, $\left.J=21.5 \mathrm{~Hz} ; \mathrm{Ar}-\mathrm{F}\right)$, 128.9 (d, J = 8.1 Hz; Ar-F), 136.5 (d, J = 3.2 Hz; Ar-F), 162.4 (d, $J=246.3 \mathrm{~Hz} ; \mathrm{Ar}-\mathrm{F}), 176.0$ (COOR).

4-Methylphenylferrocenylmethyl Valerate (1c): This compound was obtained from 4-methylphenylferrocenemethanol (0.5 g, $1.63 \mathrm{mmol})$, pyridine $(0.5 \mathrm{~g}, 6.32 \mathrm{mmol})$, and valeroyl chloride $(0.39 \mathrm{~g}, 3.23 \mathrm{~mol})$; yield $0.37 \mathrm{~g}, 58.3 \%$. $\left.{ }^{1} \mathrm{H} \mathrm{NMR} \mathrm{(400} \mathrm{MHz,} \mathrm{CDCl}{ }_{3}, 25^{\circ} \mathrm{C}\right): \delta / \mathrm{ppm}=0.90(\mathrm{t}, J=7.3 \mathrm{~Hz}, 3 \mathrm{H}$; $\mathrm{CH}_{3} \mathrm{CH}_{2} \mathrm{CH}_{2} \mathrm{CH}_{2} \mathrm{COOR}$ ), 1.32-1.36 (m, 2H; $\mathrm{CH}_{3} \mathrm{CH}_{2} \mathrm{CH}_{2} \mathrm{CH}_{2} \mathrm{COOR}$ ), 1.61-1.65 (m, 2H; $\mathrm{CH}_{3} \mathrm{CH}_{2} \mathrm{CH}_{2} \mathrm{CH}_{2} \mathrm{COOR}$ ), 2.33-2.38 (m, 5H; $\left.\mathrm{CH}_{3} \mathrm{CH}_{2} \mathrm{CH}_{2} \mathrm{CH}_{2} \mathrm{COOR}+\mathrm{CH}_{3} \mathrm{Ar}\right), 4.00-4.30\left(\mathrm{~m}, 4 \mathrm{H} ; \mathrm{C}_{5} \mathrm{H}_{4}\right)$, $4.09\left(\mathrm{~s}, 5 \mathrm{H} ; \mathrm{C}_{5} \mathrm{H}_{5}\right), 6.71(\mathrm{~s}, 1 \mathrm{H} ; \mathrm{ArCHFc}), 7.14(\mathrm{~d}, J=8.0 \mathrm{~Hz}$, $2 \mathrm{H} ; \mathrm{Ar}), 7.29$ (d, $J=8.0 \mathrm{~Hz}, 2 \mathrm{H}$; Ar). ${ }^{13} \mathrm{C}$ NMR $(100 \mathrm{MHz}$, 
$\left.\mathrm{CDCl}_{3}, 25^{\circ} \mathrm{C}\right): \delta / \mathrm{ppm}=13.9\left(\mathrm{CH}_{3} \mathrm{CH}_{2} \mathrm{CH}_{2} \mathrm{CH}_{2} \mathrm{COOR}\right), 21.3\left(\mathrm{CH}_{3} \mathrm{Ar}\right)$, $22.4\left(\mathrm{CH}_{3} \mathrm{CH}_{2} \mathrm{CH}_{2} \mathrm{CH}_{2} \mathrm{COOR}\right), 27.2\left(\mathrm{CH}_{3} \mathrm{CH}_{2} \mathrm{CH}_{2} \mathrm{CH}_{2} \mathrm{COOR}\right), 34.5$ $\left(\mathrm{CH}_{3} \mathrm{CH}_{2} \mathrm{CH}_{2} \mathrm{CH}_{2} \mathrm{COOR}\right), 67.6,67.7,68.2,68.3\left(\mathrm{C}_{5} \mathrm{H}_{4}\right), 69.0$ $\left(\mathrm{C}_{5} \mathrm{H}_{5}\right), 74.0$ (ArCHFc), 88.4 (Fc), 127.2, 129.0, 137.5, 137.7 (Ar), 172.9 (COOR).

4-tert-butylphenylferrocenylmethyl Valerate (2c): This compound was obtained from 4-tert-butylphenylferrocenemethanol $(0.5 \mathrm{~g}, 1.44 \mathrm{mmol})$, pyridine $(0.5 \mathrm{~g}$, $6.32 \mathrm{mmol})$, and valeroyl chloride $(0.35 \mathrm{~g}, 2.90 \mathrm{~mol})$; yield $0.40 \mathrm{~g}, 73.6 \% .{ }^{1} \mathrm{H} \mathrm{NMR}\left(400 \mathrm{MHz}, \mathrm{CDCl}_{3}, 25^{\circ} \mathrm{C}\right): \delta / \mathrm{ppm}=$ $0.91\left(\mathrm{t}, J=7.3 \mathrm{~Hz}, 3 \mathrm{H} ; \mathrm{CH}_{3} \mathrm{CH}_{2} \mathrm{CH}_{2} \mathrm{CH}_{2} \mathrm{COOR}\right.$ ), 1.31-1.38 (m, $\left.11 \mathrm{H} ; \mathrm{CH}_{3} \mathrm{CH}_{2} \mathrm{CH}_{2} \mathrm{CH}_{2} \mathrm{COOR}+\left(\mathrm{CH}_{3}\right)_{3} \mathrm{CAr}\right), 1.61-1.67$ (m, $2 \mathrm{H} ; \mathrm{CH}_{3} \mathrm{CH}_{2} \mathrm{CH}_{2} \mathrm{CH}_{2} \mathrm{COOR}$ ), $2.38(\mathrm{t}, J=7.4 \mathrm{~Hz}, 2 \mathrm{H}$; $\mathrm{CH}_{3} \mathrm{CH}_{2} \mathrm{CH}_{2} \mathrm{CH}_{2} \mathrm{COOR}$ ), 4.04-4.32 (m, 4H; $\left.\mathrm{C}_{5} \mathrm{H}_{4}\right), 4.10(\mathrm{~s}, 5 \mathrm{H}$; $\left.\mathrm{C}_{5} \mathrm{H}_{5}\right), 6.74(\mathrm{~s}, 1 \mathrm{H}$; $\mathrm{ArCHFc}), 7.31-7.36(\mathrm{~m}, 4 \mathrm{H} ; \mathrm{Ar}) .{ }^{13} \mathrm{C} \mathrm{NMR}$ $\left(100 \mathrm{MHz}, \mathrm{CDCl}_{3}, 25^{\circ} \mathrm{C}\right): \delta / \mathrm{ppm}=13.9\left(\mathrm{CH}_{3} \mathrm{CH}_{2} \mathrm{CH}_{2} \mathrm{CH}_{2} \mathrm{COOR}\right)$, $22.4\left(\mathrm{CH}_{3} \mathrm{CH}_{2} \mathrm{CH}_{2} \mathrm{CH}_{2} \mathrm{COOR}\right)$, $27.2\left(\mathrm{CH}_{3} \mathrm{CH}_{2} \mathrm{CH}_{2} \mathrm{CH}_{2} \mathrm{COOR}\right)$, $31.5\left(\left(\mathrm{CH}_{3}\right)_{3} \mathrm{CAr}, 34.5\left(\mathrm{CH}_{3} \mathrm{CH}_{2} \mathrm{CH}_{2} \mathrm{CH}_{2} \mathrm{COOR}\right), 34.7\left(\left(\mathrm{CH}_{3}\right)_{3} \mathrm{CAr}\right)\right.$, 67.6, 67.6, 68.2, $68.2\left(\mathrm{C}_{5} \mathrm{H}_{4}\right), 69.0\left(\mathrm{C}_{5} \mathrm{H}_{5}\right), 73.9$ (ArCHFC), 88.6 (Fc), 125.2, 127.0, 137.5, 150.9 (Ar), 173.0 (COOR).

4-Ethylphenylferrocenylmethyl Valerate (3c): This compound was obtained from 4-ethylphenylferrocenemethanol $(0.5 \mathrm{~g}, 1.56 \mathrm{mmol})$, pyridine $(0.5 \mathrm{~g}, 6.32 \mathrm{mmol})$, and valeroyl chloride $(0.37 \mathrm{~g}, 3.07 \mathrm{~mol})$; yield $0.48 \mathrm{~g}, 76.3 \%$. ${ }^{1} \mathrm{H} \mathrm{NMR}\left(400 \mathrm{MHz}, \mathrm{CDCl}_{3}, 25^{\circ} \mathrm{C}\right): \delta / \mathrm{ppm}=0.92(\mathrm{t}, J=7.3 \mathrm{~Hz}$, $3 \mathrm{H} ; \mathrm{CH}_{3} \mathrm{CH}_{2} \mathrm{CH}_{2} \mathrm{CH}_{2} \mathrm{COOR}$ ), 1.24 (t, J=7.6 Hz, 3H; $\mathrm{CH}_{3} \mathrm{CH}_{2} \mathrm{Ar}$ ), 1.32-1.38 (m, 2H; $\left.\mathrm{CH}_{3} \mathrm{CH}_{2} \mathrm{CH}_{2} \mathrm{CH}_{2} \mathrm{COOR}\right), 1.61-1.67$ (m, $2 \mathrm{H} ; \mathrm{CH}_{3} \mathrm{CH}_{2} \mathrm{CH}_{2} \mathrm{CH}_{2} \mathrm{COOR}$ ), $2.37(\mathrm{t}, J=7.3 \mathrm{~Hz}, 2 \mathrm{H}$; $\mathrm{CH}_{3} \mathrm{CH}_{2} \mathrm{CH}_{2} \mathrm{CH}_{2} \mathrm{COOR}$ ), 2.65 (q, $J=7.6 \mathrm{~Hz}, 2 \mathrm{H} ; \mathrm{CH}_{3} \mathrm{CH}_{2} \mathrm{Ar}$ ), 4.03-4.32 (m, 4H; $\left.\mathrm{C}_{5} \mathrm{H}_{4}\right), 4.11\left(\mathrm{~s}, 5 \mathrm{H} ; \mathrm{C}_{5} \mathrm{H}_{5}\right), 6.74$ (s, $1 \mathrm{H}$; $\operatorname{ArCHFc}), 7.18(\mathrm{~d}, J=8.0 \mathrm{~Hz}, 2 \mathrm{H} ; \mathrm{Ar}), 7.33$ (d, $J=8.1 \mathrm{~Hz}, 2 \mathrm{H}$; $\mathrm{Ar}) .{ }^{13} \mathrm{C} \mathrm{NMR}\left(100 \mathrm{MHz}, \mathrm{CDCl}_{3}, 25^{\circ} \mathrm{C}\right)$ : $\delta / p p m=13.8\left(\mathrm{CH}_{3} \mathrm{CH}_{2} \mathrm{CH}_{2} \mathrm{CH}_{2} \mathrm{COOR}\right), 15.5\left(\mathrm{CH}_{3} \mathrm{CH}_{2} \mathrm{Ar}\right)$, $22.3\left(\mathrm{CH}_{3} \mathrm{CH}_{2} \mathrm{CH}_{2} \mathrm{CH}_{2} \mathrm{COOR}\right), 27.1\left(\mathrm{CH}_{3} \mathrm{CH}_{2} \mathrm{CH}_{2} \mathrm{CH}_{2} \mathrm{COOR}\right)$, $28.6\left(\mathrm{CH}_{3} \mathrm{CH}_{2} \mathrm{Ar}\right)$, 34.4 $\left(\mathrm{CH}_{3} \mathrm{CH}_{2} \mathrm{CH}_{2} \mathrm{CH}_{2} \mathrm{COOR}\right), 67.5,67.6$, 68.1, $68.2\left(\mathrm{C}_{5} \mathrm{H}_{4}\right), 68.8\left(\mathrm{C}_{5} \mathrm{H}_{5}\right), 73.9(\mathrm{ArCHFc}), 88.4(\mathrm{Fc})$, 127.2, 127.7, 137.6, 143.9 ( $\mathrm{Ar}), 172.8$ (COOR).

4-Fluorophenylferrocenylmethyl Valerate (4c): This compound was obtained from 4-fluorophenylferrocenemethanol $(0.5 \mathrm{~g}, 1.61 \mathrm{mmol})$, pyridine $(0.5 \mathrm{~g}, 6.32 \mathrm{mmol})$, and valeroyl chloride $(0.40 \mathrm{~g}, 3.32 \mathrm{mmol})$; yield $0.40 \mathrm{~g}$, $62.7 \%$. ${ }^{1} \mathrm{H}$ NMR $\left(400 \mathrm{MHz}, \mathrm{CDCl}_{3}, 25^{\circ} \mathrm{C}\right): \delta / \mathrm{ppm}=0.90(\mathrm{t}$, $\left.J=7.3 \mathrm{~Hz}, 3 \mathrm{H} ; \mathrm{CH}_{3} \mathrm{CH}_{2} \mathrm{CH}_{2} \mathrm{CH}_{2} \mathrm{COOR}\right), 1.30-1.36(\mathrm{~m}, 2 \mathrm{H}$; $\mathrm{CH}_{3} \mathrm{CH}_{2} \mathrm{CH}_{2} \mathrm{CH}_{2} \mathrm{COOR}$ ), 1.61-1.64 (m, 2H; $\mathrm{CH}_{3} \mathrm{CH}_{2} \mathrm{CH}_{2} \mathrm{CH}_{2} \mathrm{COOR}$ ), 2.36 (t, J = 7.5 Hz, 2H; $\mathrm{CH}_{3} \mathrm{CH}_{2} \mathrm{CH}_{2} \mathrm{CH}_{2} \mathrm{COOR}$ ), 3.98-4.30 (m, $\left.4 \mathrm{H} ; \mathrm{C}_{5} \mathrm{H}_{4}\right), 4.10\left(\mathrm{~s}, 5 \mathrm{H} ; \mathrm{C}_{5} \mathrm{H}_{5}\right), 6.72(\mathrm{~s}, 1 \mathrm{H} ; \mathrm{ArCHFc}), 7.00-7.39$ (m, 4H; Ar). ${ }^{19} \mathrm{~F}$ NMR $\left(376 \mathrm{MHz}, \mathrm{CDCl}_{3}, 25{ }^{\circ} \mathrm{C}\right): \delta / \mathrm{ppm}=$ $-114.1(\mathrm{~m}) .{ }^{13} \mathrm{C} \mathrm{NMR}\left(100 \mathrm{MHz}, \mathrm{CDCl}_{3}, 25^{\circ} \mathrm{C}\right): \delta / \mathrm{ppm}=13.86$ ( $\left.\mathrm{CH}_{3} \mathrm{CH}_{2} \mathrm{CH}_{2} \mathrm{CH}_{2} \mathrm{COOR}\right), 22.4\left(\mathrm{CH}_{3} \mathrm{CH}_{2} \mathrm{CH}_{2} \mathrm{CH}_{2} \mathrm{COOR}\right), 27.2$ ( $\left.\mathrm{CH}_{3} \mathrm{CH}_{2} \mathrm{CH}_{2} \mathrm{CH}_{2} \mathrm{COOR}\right)$, $34.4\left(\mathrm{CH}_{3} \mathrm{CH}_{2} \mathrm{CH}_{2} \mathrm{CH}_{2} \mathrm{COOR}\right)$, 67.4, 67.6, 68.3, $68.5\left(\mathrm{C}_{5} \mathrm{H}_{4}\right), 69.0\left(\mathrm{C}_{5} \mathrm{H}_{5}\right), 73.5$ (ArCHFC), 88.0 (Fc),
115.2 (d, $J=21.5 \mathrm{~Hz} ; \mathrm{Ar}-\mathrm{F}$ ), 129.1 (d, J=8.1 Hz; Ar-F), 136.4 (d, $J=3.0 \mathrm{~Hz} ; \mathrm{Ar}-\mathrm{F}$ ), 162.4 (d, $J=246.3 \mathrm{~Hz} ; \mathrm{Ar}-\mathrm{F}$ ), 172.9 (COOR).

4-Methylphenylferrocenylmethyl Isovalerate (1d): This compound was obtained from 4-methylphenylferrocenemethanol (0.5 g, $1.63 \mathrm{mmol})$, pyridine $(0.5 \mathrm{~g}, 6.32 \mathrm{mmol})$, and isovaleryl chloride $(0.39 \mathrm{~g}, 3.23 \mathrm{~mol})$; yield $0.37 \mathrm{~g}$, $58.3 \% .{ }^{1} \mathrm{H} \mathrm{NMR}\left(400 \mathrm{MHz}, \mathrm{CDCl}_{3}, 25^{\circ} \mathrm{C}\right): \delta / \mathrm{ppm}=0.92-0.94$ $\left(\mathrm{d}+\mathrm{d}, J=3.1 \mathrm{~Hz}+J=3.0 \mathrm{~Hz}, 3 \mathrm{H}+3 \mathrm{H} ;\left(\mathrm{CH}_{3}\right)_{2} \mathrm{CHCH}_{2} \mathrm{COOR}\right)$, 2.09-2.16 (m, $\left.1 \mathrm{H} ;\left(\mathrm{CH}_{3}\right)_{2} \mathrm{CHCH}_{2} \mathrm{COOR}\right), 2.23(\mathrm{~d}, J=7.1 \mathrm{~Hz}$, $\left.2 \mathrm{H} ;\left(\mathrm{CH}_{3}\right)_{2} \mathrm{CHCH}_{2} \mathrm{COOR}\right), 2.34$ (s, 3H; $\left.\mathrm{CH}_{3} \mathrm{Ar}\right), 3.99-4.30$ (m, $\left.4 \mathrm{H} ; \mathrm{C}_{5} \mathrm{H}_{4}\right), 4.09\left(\mathrm{~s}, 5 \mathrm{H} ; \mathrm{C}_{5} \mathrm{H}_{5}\right), 6.71(\mathrm{~s}, 1 \mathrm{H} ; \mathrm{ArCHFc}), 7.15$ $(\mathrm{d}, J=8.0 \mathrm{~Hz}, 2 \mathrm{H} ; \mathrm{Ar}), 7.30(\mathrm{~d}, J=7.9 \mathrm{~Hz}, 2 \mathrm{H} ; \mathrm{Ar}) .{ }^{13} \mathrm{C} \mathrm{NMR}$ $\left(100 \mathrm{MHz}, \mathrm{CDCl}_{3}, 25^{\circ} \mathrm{C}\right): \delta / \mathrm{ppm}=21.3\left(\mathrm{CH}_{3} \mathrm{Ar}\right), 22.6+$ $22.6\left(\left(\mathrm{CH}_{3}\right)_{2} \mathrm{CHCH}_{2} \mathrm{COOR}\right), 25.9\left(\left(\mathrm{CH}_{3}\right)_{2} \mathrm{CHCH}_{2} \mathrm{COOR}\right), 43.8$ $\left(\left(\mathrm{CH}_{3}\right)_{2} \mathrm{CHCH}_{2} \mathrm{COOR}\right), 67.6,67.9,68.2,68.3\left(\mathrm{C}_{5} \mathrm{H}_{4}\right), 69.0$ $\left(\mathrm{C}_{5} \mathrm{H}_{5}\right), 74.1$ (ArCHFc), 88.4 (Fc), 127.3, 129.0, 137.4, 137.8 (Ar), 172.3 (COOR).

4-tert-butylphenylferrocenylmethyl Isovalerate (2d): This compound was obtained from 4-tert-butylphenylferrocenemethanol $(0.5 \mathrm{~g}, 1.44 \mathrm{mmol})$, pyridine $(0.5 \mathrm{~g}, 6.32$ $\mathrm{mmol})$, and isovaleryl chloride $(0.26 \mathrm{~g}, 2.16 \mathrm{~mol})$; yield $0.37 \mathrm{~g}, 58.3 \% .{ }^{1} \mathrm{H}$ NMR $\left(400 \mathrm{MHz}, \mathrm{CDCl}_{3}, 25^{\circ} \mathrm{C}\right): \delta / \mathrm{ppm}=$ $0.93-0.95(\mathrm{~d}+\mathrm{d}, J=3.0 \mathrm{~Hz}+J=3.0 \mathrm{~Hz}, 3 \mathrm{H}+3 \mathrm{H}$; $\left.\left(\mathrm{CH}_{3}\right)_{2} \mathrm{CHCH}_{2} \mathrm{COOR}\right), 1.30\left(\mathrm{~s}, 9 \mathrm{H} ;\left(\mathrm{CH}_{3}\right)_{3} \mathrm{CAr}\right), 2.10-2.17$ (m, $\left.1 \mathrm{H} ;\left(\mathrm{CH}_{3}\right)_{2} \mathrm{CHCH}_{2} \mathrm{COOR}\right), 2.24(\mathrm{~d}, J=7.0 \mathrm{~Hz}, 2 \mathrm{H}$; $\left.\left(\mathrm{CH}_{3}\right)_{2} \mathrm{CHCH}_{2} \mathrm{COOR}\right), 4.02-4.31\left(\mathrm{~m}, 4 \mathrm{H} ; \mathrm{C}_{5} \mathrm{H}_{4}\right), 4.10(\mathrm{~s}, 5 \mathrm{H}$; $\left.\mathrm{C}_{5} \mathrm{H}_{5}\right), 6.74(\mathrm{~s}, 1 \mathrm{H} ; \operatorname{ArCHFc}), 7.31-7.36(\mathrm{~m}, 4 \mathrm{H} ; \mathrm{Ar})$. ${ }^{13} \mathrm{C} \mathrm{NMR}\left(100 \mathrm{MHz}, \mathrm{CDCl}_{3}, 25{ }^{\circ} \mathrm{C}\right): \delta / \mathrm{ppm}=22.6+$ $22.6\left(\left(\mathrm{CH}_{3}\right)_{2} \mathrm{CHCH}_{2} \mathrm{COOR}\right), 25.8\left(\left(\mathrm{CH}_{3}\right)_{2} \mathrm{CHCH}_{2} \mathrm{COOR}\right), 31.5$ $\left.\left.\left(\mathrm{CH}_{3}\right)_{3} \mathrm{CAr}\right), 34.7\left(\mathrm{CH}_{3}\right)_{3} \mathrm{CAr}\right), 43.8\left(\left(\mathrm{CH}_{3}\right)_{2} \mathrm{CHCH}_{2} \mathrm{COOR}\right), 67.6$, 67.7, 68.2, $68.2\left(\mathrm{C}_{5} \mathrm{H}_{4}\right), 69.0\left(\mathrm{C}_{5} \mathrm{H}_{5}\right), 73.9(\mathrm{ArCHFc}), 88.5(\mathrm{Fc})$, 125.2, 127.0, 137.4, 150.8 (Ar), 172.3 (COOR).

4-Ethylphenylferrocenylmethyl Isovalerate (3d): This compound was obtained from 4-ethylphenylferrocenemethanol (0.5 g, $1.56 \mathrm{mmol})$, pyridine $(0.5 \mathrm{~g}, 6.32 \mathrm{mmol})$, and isovaleryl chloride $(0.28 \mathrm{~g}, 2.32 \mathrm{~mol})$; yield $0.28 \mathrm{~g}, 44.2 \%$. ${ }^{1} \mathrm{H} \mathrm{NMR}\left(400 \mathrm{MHz}, \mathrm{CDCl}_{3}, 25^{\circ} \mathrm{C}\right): \delta / \mathrm{ppm}=0.93-0.94(\mathrm{~d}+\mathrm{d}$, $\left.J=3.1 \mathrm{~Hz}+J=3.1 \mathrm{~Hz}, 3 \mathrm{H}+3 \mathrm{H} ;\left(\mathrm{CH}_{3}\right)_{2} \mathrm{CHCH}_{2} \mathrm{COOR}\right), 1.23(\mathrm{t}, J=$ $\left.7.6 \mathrm{~Hz}, 3 \mathrm{H} ; \mathrm{CH}_{3} \mathrm{CH}_{2} \mathrm{Ar}\right), 2.09-2.16\left(\mathrm{~m}, 1 \mathrm{H} ;\left(\mathrm{CH}_{3}\right)_{2} \mathrm{CHCH}_{2} \mathrm{COOR}\right)$, 2.24 (d, $J=7.1 \mathrm{~Hz}, 2 \mathrm{H} ;\left(\mathrm{CH}_{3}\right)_{2} \mathrm{CHCH}_{2} \mathrm{COOR}$ ), 2.64 (q, $J=7.6$ $\mathrm{Hz}, 2 \mathrm{H} ; \mathrm{CH}_{3} \mathrm{CH}_{2} \mathrm{Ar}$ ), 4.00-4.31 (m, 4H; $\left.\mathrm{C}_{5} \mathrm{H}_{4}\right), 4.10(\mathrm{~s}, 5 \mathrm{H}$; $\left.\mathrm{C}_{5} \mathrm{H}_{5}\right), 6.67(\mathrm{~s}, 1 \mathrm{H} ; \operatorname{ArCHFc}), 7.17(\mathrm{~d}, 2 \mathrm{H}, J=8.1 \mathrm{~Hz} ; \mathrm{Ar}), 7.32$ $(\mathrm{d}, 2 \mathrm{H}, J=8.0 \mathrm{~Hz}$; $\mathrm{Ar}) .{ }^{13} \mathrm{C} \mathrm{NMR}\left(100 \mathrm{MHz}, \mathrm{CDCl}_{3}, 25^{\circ} \mathrm{C}\right)$ : $\delta / p p m=15.6\left(\mathrm{CH}_{3} \mathrm{CH}_{2} \mathrm{Ar}\right), 22.6+22.6\left(\left(\mathrm{CH}_{3}\right)_{2} \mathrm{CHCH}_{2} \mathrm{COOR}\right)$, $25.8 \quad\left(\left(\mathrm{CH}_{3}\right)_{2} \mathrm{CHCH}_{2} \mathrm{COOR}\right), \quad 28.7 \quad\left(\mathrm{CH}_{3} \mathrm{CH}_{2} \mathrm{Ar}\right), \quad 43.8$ $\left(\left(\mathrm{CH}_{3}\right)_{2} \mathrm{CHCH}_{2} \mathrm{COOR}\right), 67.6,67.8,68.2,68.3\left(\mathrm{C}_{5} \mathrm{H}_{4}\right), 69.0$ $\left(\mathrm{C}_{5} \mathrm{H}_{5}\right), 74.1$ (ArCHFc), 88.4 (Fc), 127.3, 127.8, 137.7, 144.0 (Ar), 172.3 (COOR). 
4-Fluorophenylferrocenylmethyl Isovalerate (4d): This compound was obtained from 4-fluorophenylferrocenemethanol $(0.5 \mathrm{~g}, 1.61 \mathrm{mmol})$, pyridine $(0.5 \mathrm{~g}, 6.32 \mathrm{mmol})$, and isovaleryl chloride $(0.29 \mathrm{~g}, 2.41 \mathrm{mmol})$; yield $0.37 \mathrm{~g}$, $58.4 \% .{ }^{1} \mathrm{H} \mathrm{NMR}\left(400 \mathrm{MHz}, \mathrm{CDCl}_{3}, 25^{\circ} \mathrm{C}\right): \delta / \mathrm{ppm}=0.92-0.94$ $\left(\mathrm{d}+\mathrm{d}, J=3.0 \mathrm{~Hz}+J=2.8 \mathrm{~Hz}, 3 \mathrm{H}+3 \mathrm{H} ;\left(\mathrm{CH}_{3}\right)_{2} \mathrm{CHCH}_{2} \mathrm{COOR}\right)$, 2.10-2.15 (m, $\left.1 \mathrm{H} ;\left(\mathrm{CH}_{3}\right)_{2} \mathrm{CHCH}_{2} \mathrm{COOR}\right), 2.24(\mathrm{~d}, J=7.1 \mathrm{~Hz}$, $2 \mathrm{H}$; $\left.\left(\mathrm{CH}_{3}\right)_{2} \mathrm{CHCH}_{2} \mathrm{COOR}\right), 3.97-4.30\left(\mathrm{~m}, 4 \mathrm{H} ; \mathrm{C}_{5} \mathrm{H}_{4}\right), 4.10$ (s, $\left.5 \mathrm{H} ; \mathrm{C}_{5} \mathrm{H}_{5}\right), 6.72(\mathrm{~s}, 1 \mathrm{H} ; \mathrm{ArCHFc}), 7.01-7.40(\mathrm{~m}, 4 \mathrm{H} ; \mathrm{Ar}) .{ }^{19} \mathrm{~F}$ NMR (376 MHz, $\left.\mathrm{CDCl}_{3}, 25{ }^{\circ} \mathrm{C}\right): \delta / \mathrm{ppm}=-114.1(\mathrm{~m})$. ${ }^{13} \mathrm{C} \mathrm{NMR}\left(100 \mathrm{MHz}, \mathrm{CDCl}_{3}, 25^{\circ} \mathrm{C}\right): \delta / \mathrm{ppm}=22.5+22.6$ (( $\left.\left.\mathrm{CH}_{3}\right)_{2} \mathrm{CHCH}_{2} \mathrm{COOR}\right), \quad 25.9 \quad\left(\left(\mathrm{CH}_{3}\right)_{2} \mathrm{CHCH}_{2} \mathrm{COOR}\right), \quad 43.7$ (( $\left.\left.\mathrm{CH}_{3}\right)_{2} \mathrm{CHCH}_{2} \mathrm{COOR}\right), 67.4,67.8,68.4,68.5\left(\mathrm{C}_{5} \mathrm{H}_{4}\right), 69.0$ $\left(\mathrm{C}_{5} \mathrm{H}_{5}\right), 73.5$ (ArCHFc), $88.0(\mathrm{Fc}), 115.2(\mathrm{~d}, J=21.5 \mathrm{~Hz} ; \mathrm{Ar}-\mathrm{F})$, 129.1 (d, J = 8.1 Hz; Ar-F), 136.3 (d, J=3.3 Hz; Ar-F), 162.4 (d, J = 246.4 Hz; Ar-F), 172.2 (COOR).

Kinetic Methods: Solvolysis rate constants were measured titrimetrically by means of TIM 856 titration manager (Radiometer Analytical SAS Villeurbanne Cedex, France), using a Red Rod $\mathrm{Ag} / \mathrm{AgCl}$ combined $\mathrm{pH}$ electrode. Typically, 20-50 mg of the carboxylates were dissolved in $0.10-0.20$ $\mathrm{mL}$ of dichloromethane, and injected into the solvent that was thermostated at the required temperature $\left( \pm 0.01^{\circ} \mathrm{C}\right)$. The liberated acid was continuously titrated at $\mathrm{pH}=7.00$ 7.80 by using a $0.016 \mathrm{M}$ or $0.032 \mathrm{M}$ solution of sodium hydroxide in appropriate solvent. Individual rate constants were obtained by the least-squares fitting of data to the firstorder kinetic equation for three to four half-lives. The rate constants were averaged from at least three measurements.

Acknowledgment. The authors gratefully acknowledge financial support of this research by the Croatian Science Foundation (under the project number IP-1021).

Supplementary Information. Supporting information to the paper is attached to the electronic version of the article at: https://doi.org/10.5562/cca3553.

PDF files with attached documents are best viewed with Adobe Acrobat Reader which is free and can be downloaded from Adobe's web site.

\section{REFERENCES}

[1] [a] M. B. Smith, J. March, March's Advanced Organic Chemistry: Reactions, Mechanisms and Structure, 6th ed., A John Wiley \& Sons, Inc.; Hoboken, New Jersey, 2007. [b] T. H. Lowry, K. S. Richardson, Mechanism and Theory in Organic Chemistry, $3^{\text {rd }}$ ed., Harper \& Row, New York, 1987.
[2] [a] B. Denegri, A. Streiter, S. Jurić, A. R. Ofial, O. Kronja, H. Mayr, Chem.-Eur. J. 2006, 12, 1648-1656.

https://doi.org/10.1002/chem.200500845

[b] Correction: B. Denegri, A. Streiter, S. Jurić, A. R. Ofial, O. Kronja, H. Mayr, Chem.-Eur. J. 2006, 12, 5415-5415.

https://doi.org/10.1002/chem.200690066

[c] B. Denegri, A. R.Ofial, S. Jurić, A. Streiter, O. Kronja, H. Mayr, Chem.-Eur. J. 2006, 12, 1657-1666.

https://doi.org/10.1002/chem.200500847

[3] [a] N. Streidl, B. Denegri, O. Kronja, H. Mayr, Acc. Chem. Res. 2010, 43, 1537-1549.

https://doi.org/10.1021/ar100091m

[b] B. Denegri, M. Matić, O. Kronja, Synthesis 2017, 49, 3422-3432.

https://doi.org/10.1055/s-0036-1590792

[4] for some selected examples see: [a] S. Jurić, B. Denegri, O. Kronja, J. Org. Chem. 2010, 75, 38513854. https://doi.org/10.1021/jo100327c

[b] S. Jurić, B. Denegri, O. Kronja, J. Phys. Org. Chem. 2012, 25, 147-152.

https://doi.org/10.1002/poc.1886

[c] S. Jurić, O. Kronja, J. Phys. Org. Chem. 2015, 28, 314-319. https://doi.org/10.1002/poc.3412

[d] S. Jurić, T. Portolan, O. Kronja, Croat. Chem. Acta 2016, 89, 65-70.

https://doi.org/10.5562/cca2822

[e] M. Matić, B. Denegri, S. Jurić, O. Kronja, Croat. Chem. Acta 2017, 90, 571-581.

https://doi.org/10.5562/cca3283

[5] M. Horn, C. Metz, H. Mayr, Eur. J. Org. Chem. 2011, 6476-6485.

https://doi.org/10.1002/ejoc.201100912

[6] M. Marijan, S. Jurić, Z. Mihalić, O. Kronja, Eur. J. Org. Chem. 2019, 537-546.

https://doi.org/10.1002/ejoc.201801133

[7] [a] M. Matić, B. Denegri, O. Kronja, Eur. J. Org. Chem. 2014, 1477-1486.

https://doi.org/10.1002/ejoc.201301574

[b] B. Denegri, O. Kronja, Croat. Chem. Acta 2010, 83, 223-226.

[8] [a] M. Rousch, M. Vogel, H. Rosenberg, J. Org. Chem. 1957, 22, 903-906.

https://doi.org/10.1021/jo01359a012

[b] R. L. Schaaf, J. Org. Chem. 1962, 27, 107-111.

https://doi.org/10.1021/jo01048a027

[9] K. Schlögl, Monatsh. Chem. 1957, 88, 601-621. https://doi.org/10.1007/BF00901345 